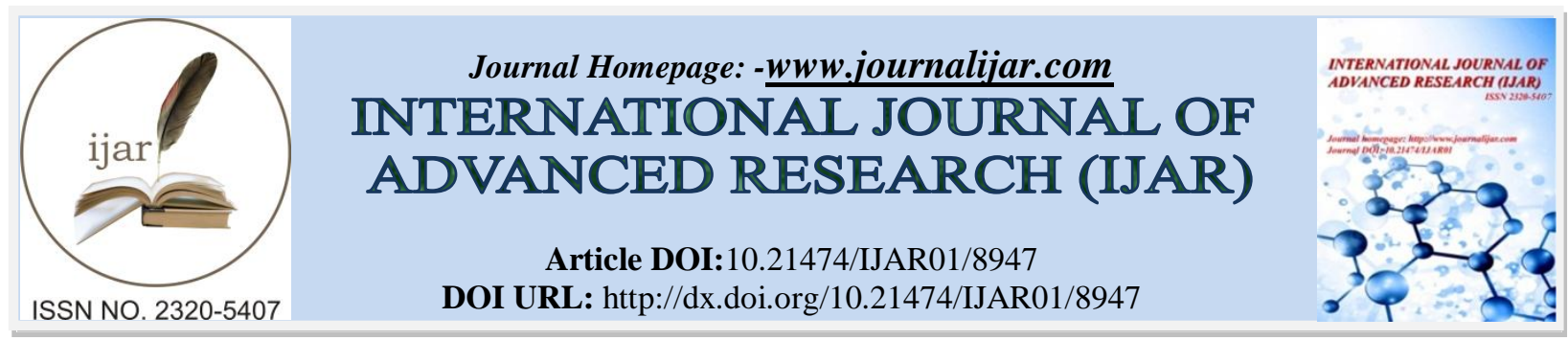

RESEARCH ARTICLE

\title{
GYNECOLOGICAL ISSUES IN ADOLESCENT GIRLS AND EVALUATION OF CASES OF PUBERTY MENORRHAGIA REQUIRING INDOOR ADMISSION.
}

DR. Swathi Pai and Dr. Sujata Singh.

Department of Obstetrics and Gynecology, S C B medical college, Cuttack.

\section{Manuscript Info}

Manuscript History

Received: 22 February 2019

Final Accepted: 24 March 2019

Published: April 2019

\section{Abstract}

\section{Introduction:-}

According to World Health Organization, adolescence is defined as the age between 10 and 19 years ${ }^{[1]}$ and is a transitional stage between childhood and adulthood, during which major physical and mental changes occur. The complexity of gynecological problems in adolescents makes them special not just because of the clinical challenges but also the associated psychological factors which play a key role in the transition between childhood and womanhood.

Adolescents have the lowest mortality among the different age groups so have received low priority. Many girls in this age with menstrual problems never present to their family doctor or Gynecologist. Embarrassment about discussing menstruation, fear of disease, and ignorance and lack of knowledge regarding the availability of health services will lead to late presentation to the consultant.

\section{Adolescence can be divided into three sub phases}

1. Early adolescence (10-14 years): characterized by the onset of puberty and transition out of childhood.

2. Middle adolescence (15-17 years): Identification with group, indicated by wearing of particular clothing, listening to same music, adopting similar verbal phrases.

3. Late adolescence (18-19 years): Transition into adult roles indicated by responsible and mature approach to one's personal and intimate relationships. ${ }^{[2]}$

\section{Aims And Objective:-}

1. The aim of the study was to evaluate gynecological problems in adolescent girls attending the OPD

2. To assess the causes and evaluate cases of puberty menorrhagia which required hospitalization

\section{Methods:-}

1. The study was conducted in S.C.B Medical College

2. A prospective cross-sectional study

3. All adolescent girls attending the O\&G OPD between November 2016 to May 2017 were included in this study.

4. Statistical analysis was done using Microsoft Excel

Corresponding Author:- Dr. Sujata Singh.

Address:-Department of Obstetrics and Gynecology, S C B medical college, Cuttack. 


\section{Results:-}

Out of the total number of patient 23,453 attending gynecological OPD 745 patients $(3.17 \%)$ belonged to the adolescent age group i.e. 10-19 years. Maximum adolescent girls attending Gynecological OPD had ages in the range $10-14$ years age group.

We had 313 (42.01\%)patients in the age group of 10-14(early adolescent group), 281(37.71\%)patients belonged to the age group of 15-17 years(middle adolescent group) and 151(20.26\%)in the age group of 18-19years (late adolescent group).

Majority of adolescent girls were under-weight with BMI $<18.5$ (462 out of 745: 62.01\%). This could be due to poverty and illiteracy prevalent in our country.

Increasing trends of obesity were also seen (120 out of 745: 16\%).

Maximum patients attained menarche between the age group of 15-16 years which was 538 (72.21\%) patients.

Gynecological Problems Amongst Adolescent Girls

MENSTRUAL DISORDERS were observed in 589 (79.06\%) patients and was the most common problem seeking medical attention.

VULVOVAGINAL CONDITIONS was the second most common seen in 125(16.77\%) patients.

TEENAGE PREGNANCY accounted to $2.55 \%$ (19) patients.

UTI was reported in 12 patients accounting to $1.61 \%$.

\section{Menstrual disorders:}

Majority of adolescent girls suffered from menstrual disorders, i.e. 589 out 0 f $745 ; 79.06 \%$ from amenorrhea to menorrhagia. Among the patients suffering from secondary amenorrhea (73) and oligomenorrhoea (193) 102 were ultimately diagnosed to be cases of polycystic ovarian disease (PCOD) $13.69 \%$. Associated problems like obesity were also present in half of them. Dysmenorrhea was the most common complain seen in 208(35.31\%) patients. Hypomenorrhea and oligomenorrhea observed in 193(32.76\%) patients. Menorrhagia and metrorrhagia accounting to $112(19.01 \%)$ patients. Primary Amenorrhea was seen in 3 patients accounting to $0.50 \%$ and secondary amenorrhea was seen in 73 patients accounting to $12.39 \%$.

\section{Menorrhagia}

Out of 112 patients suffering from menorrhagia and metrorrhagia 14 patients required admission for anemia correction and management of menorrhagia. Majority of the patients on investigating the causes of menorrhagia, in 107 out of 112 girls (95.53\%) no cause could be ascertained, i.e. they suffered from Dysfunctional Uterine Bleeding .First line treatment was tranexamic acid/mefenamic acid and NSAIDS during menstrual cycles.

Puberty Menorrhagia

$1.87 \%$ i.e. 14 out of 745 patients required hospitalization

\begin{tabular}{|l|l|l|}
\hline Causes & Number & percentage \\
\hline Anovulatory DUB & 11 & 78.57 \\
\hline HYPOTHYROIDSM & 2 & 14.28 \\
\hline ITP & 1 & 7.14 \\
\hline VON WILLEBRAND DISEASE & 0 & 0 \\
\hline TOTAL & 14 & 100 \\
\hline
\end{tabular}

Table 1:-Causes Of Puberty Menorrhagia 
Among the 14 who were hospitalized $\mathrm{Hb}$ level of $<5 \mathrm{~g} / \mathrm{dl}$ was seen in 8 patients $(57.14 \%)$, Hb level of $5-7 \mathrm{~g} / \mathrm{dl}$ was seen in 5 patients $(35.71 \%$ ) and $\mathrm{Hb}$ level of $7-9 \mathrm{~g} / \mathrm{dl}$ was seen only in 1 patient $(7.14 \%)$. An average of 3 units of blood was transfused to the patients with $\mathrm{Hb}<5$ and an average 2 units for patients with $\mathrm{Hb} 5-7 \mathrm{~g} / \mathrm{dl}$. Majority responded well to oral progesterone and antifibrinolytic agents. ITP was treated with blood transfusions, random donor platelet transfusion and tranexamic acid and prednisolone.

\section{Primary amenorrhoea:}

Among 3 patients 1 patient had Mullerian agenesis $(0.13 \%)$ and was lost to follow up. 2 patients with imperforate hymen $(0.26 \%)$

\section{Vulvovaginal conditions:}

Vulvovaginitis is inflammation of the vulval and vaginal tissues. Poor hygiene was one of the predisposing factors. Most common was white discharge: 118 patients out of 125 i.e. 94.4\%. Pelvic Inflammatory Disease was seen exclusively in married adolescent girls owing to early sexual activity i.e. 7 out 125 patients (5.6\%)

\section{Teenage Pregnancy}

was seen in 19 patients i.e. $2.55 \%$ of cases in our study.Sexual assault was seen in 3 out of $745(0.40 \%)$ of adolescent girls in our study.

\section{Urinary tract infection:}

Among 12 patients Most common complaint was burning micturition (100\%). They were treated according to urine culture sensitivity reports.

\section{Discussion:-}

Adolescent girls present with a spectrum of gynecological disorders. It's a state of transition between childhood to adulthood, menarche being the hallmark event. In our study the incidence of gynecological problem was 3.17\% which was comparable with a study conducted by Prakriti Goswami et al ${ }^{[2]}$ which was $3.33 \%$. We had $42.01 \%$ in early adolescent group,37.71\% in middle adolescent group and $20.26 \%$ in the late adolescent group.

Most common gynecological problems were menstrual disorders which was $79.06 \%$ in our study which was comparable to a study conducted by Dr. A. Srilaxmi et al ${ }^{[3]}$ which was $72.39 \%$. Menstrual disorders included dysmenorrhoea, menorrhagia, oligomenorrhoea and primary and secondary amenorrhoea. 102 patients (13.69\%)were diagnosed to have PCOS based on clinical criteria of menstrual problems, features of hyperandrogenism, and sonography findings out of 193 patients who came with oligomenorrhoea which was $14 \%$ in a study conducted by Joshi et al ${ }^{[4]}$. $1.87 \%$ i.e. 14 out of 745 patients required hospitalization in view of menorrhagia and anemia which was lower compared to study by Dr. A. Srilakshmi et al ${ }^{[3]}$ who reported $2.05 \%$. They were diagnosed to have anovulatory bleeding (78.57\%), hypo-thyroidism $(14.28 \%)$ which was much higher compared to a study conducted by Prachi et al were it was 5.8\% and ITP (7.14\%). There were 8 patients (out of 14) who had $\mathrm{Hb}$ levels $<5 \mathrm{gm} \%$ and were transfused with an average of 3 units. 5 patients had $\mathrm{Hb}$ between $5-7 \mathrm{gm} \%$ for which an average of 2 units of blood was transfused. They were also treated with tranexamic acid and norethisterone.

Leucorrhoea and infections accounted to $16.77 \%$ which was $18.66 \%$ in a study conducted by Prakriti Goswami et al. ${ }^{[2]}$

Teenage pregnancy is a common problem in developing countries like India and was seen in $2.55 \%$ adolescent girls. Pregnancy in teenagers is a problem threatening the ultimate reproductive and child health. Sexual assault was seen in 3 out of $745(0.40 \%)$ of adolescent girls in our study. Sexual assault may lead to lower self-esteem, depression, unwanted pregnancies and criminal abortions amongst adolescents. Teenage pregnancies accounted for $4.03 \%$ of $(5 / 124)$ cases in the study conducted by Goswami Sebanti et al.$^{[5]}$

UTI was $1.61 \%$ in our study who were treated according to culture and sensitivity reports.

Vulvovaginitis is inflammation of the vulval and vaginal tissues. Poor hygiene was one of the predisposing factors.Most common was white discharge: 118 patients out of 125 i.e $94.4 \%$.Leucorrhoea is a frequent and embarrassing problem, especially in low socio-economic population. Pelvic Inflammatory Disease was seen exclusively in married adolescent girls owing to early sexual activity i.e. 7 out 125 patients(5.6\%). Leucocorrhoea was seen I $10.66 \%$ patients in a study conducted by Prakriti Goswami et al. ${ }^{[2]}$ 


\section{Conclusion:-}

Menstrual problems are quite frequent and predominant in the adolescent girls so much so that it affects their school performance and attendance as well as part of their social lives.Adolescent gynecology is not a new subject. But it needs increasing awareness and further attention especially in a country like India where early marriage and child bearing are still practiced. Since the problems are specific to this group, setting up of separate adolescent clinics is desirable for efficient management.

\section{References:-}

1. World Health Organization, Internet: Website of Department of Child and Adolescent Health and Development

2. Adolescent gynaecological problems: a prospective study Prakriti Goswami1, Geeta Ahirwar2, Prabha Mishra3, Veena Agrawal4

3. Gynecological Problems in Adolescent Girls - A Clinical Study Dr. A. Sri Lakshmi1, Dr. Koushik2

4. Joshi S. Chella H. Srivastava D. Study of pubertal Menorrhagia in adolescent girls in rural setup. J. South Asian Feder. Obset. Gynaecological 2012; 4 (2): 110 -2.

5. Goswami S. Dutta R, Sengupta S.A. profile of adolescent girls with gynaecological problems J. Obst. H Gynaecological India 1990: 55(4):353-5. 